\title{
Observations of Mars and its satellites by the Mars Imaging Camera (MIC) on Planet-B
}

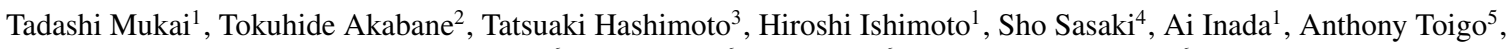 \\ Masato Nakamura ${ }^{6}$, Yutaka $\mathrm{Abe}^{6}$, Kei Kurita ${ }^{6}$, and Takeshi Imamura ${ }^{6}$ \\ ${ }^{1}$ The Graduate School of Science and Technology, Kobe University, Kobe 657-8501, Japan \\ ${ }^{2}$ Hida Observatory, Kyoto University, Kamitakara, Gifu 506-13, Japan \\ ${ }^{3}$ Institute of Space and Astronautical Science, Kanagawa 229-8510, Japan \\ ${ }^{4}$ Geological Institute, University of Tokyo, Tokyo 113, Japan \\ ${ }^{5}$ Division of Geological and Planetary Sciences, California Institute of Technology, Pasadena, CA 91125, U.S.A. \\ ${ }^{6}$ Space and Planetary Science, University of Tokyo, Tokyo 113, Japan
}

(Received August 7, 1997; Revised December 16, 1997; Accepted January 30, 1998)

\begin{abstract}
We present the specifications of the Mars Imaging Camera (MIC) on the Planet-B spin-stabilized spacecraft, and key scientific objectives of MIC observations. A non-sun-synchronous orbit of Planet-B with a large eccentricity of about 0.87 around Mars provides the opportunities (1) to observe the same region of Mars at various times of day and various solar phase angles with spatial resolution of about $60 \mathrm{~m}$ from a distance of $150 \mathrm{~km}$ altitude (at periapsis), and (2) to monitor changes of global atmospheric conditions on Mars near an apoapsis of 15 Mars radii. In addition, (3) several encounters of Planet-B with each of the two Martian satellites are scheduled during the mission lifetime of two years from October 1999 to observe their shapes and surface structures with three color filters, centered on 450,550 , and $650 \mathrm{~nm}$. (4) A search for hypothetical dust rings along the orbits of two satellites will be tried from the forward-scattering region of sunlight.
\end{abstract}

\section{Introduction}

A visual wavelength camera to be launched with the Planet-B spacecraft is called the Mars Imaging Camera (MIC). The capability of the MIC to wholy map the surface of Mars is rather poor, compared with other planned spacecraft(s) in orbit around Mars during the period of about two years from October 1999, e.g. Mars Surveyor'98 orbiter in an orbit being near sun-synchronous, polar, near circular at about $405 \mathrm{~km}$ altitude. However, the uniqueness of the MIC observations results from large elongated orbits scheduled in the mission of Planet-B, i.e. its expected orbital parameters are; periapsis of $150 \mathrm{~km}$, apoapsis of $15 R_{\mathrm{M}}\left(R_{\mathrm{M}}\right.$ denotes a radius of Mars), period of about 34 hours. Planet-B's nonsun-synchronous orbit allows it to observe the same region at various times of day and various solar phase angles. In addition, the large distance of its apoapsis provides occasions for encounters with the two Martian satellites, Phobos and Deimos. MIC specifications are noted in Section 2, and key scientific objectives of MIC are summarized in Section 3. In Section 4, a brief summary of MIC is shown.

\section{Instruments}

The size of MIC is approximately $90 \mathrm{~mm} \times 150 \mathrm{~mm} \times$ $253 \mathrm{~mm}$, and its weight is about $2.7 \mathrm{~kg}$ (see Fig. 1). Its power consumption is about $14 \mathrm{~W}$ while imaging. MIC will do optical imaging of Mars itself and the two Martian satellites with three visible color bands; 440 480 nm, 520 580 nm, and

Copy right (C) The Society of Geomagnetism and Earth, Planetary and Space Sciences (SGEPSS); The Seismological Society of Japan; The Volcanological Society of Japan; The Geodetic Society of Japan; The Japanese Society for Planetary Sciences.
630 680 nm. MIC uses tri-color linear CCDs, consisting of each $1 \times 2560$ pixel arrays. It scans the azimuth direction (orthogonal to the CCD pixels) with spacecraft spin at a rotation speed of $7.5 \mathrm{rpm}$. Its total field of view along the array is $54.2^{\circ}$ (2560 pixels in elevation direction) $\times 360^{\circ}$ (azimuth direction). However, actual image size is limited by the memory of the camera system, e.g. 1 Mbyte on-board memory which represents about $11^{\circ}$ (512 pixels in elevation direction $) \times 11^{\circ}$ (512 lines in azimuth direction at a rotation speed of $7.5 \mathrm{rpm}$ and $0.49 \mathrm{~ms}$ integration time). Resolution of the image corresponds to about 80 arc seconds in both elevation and azimuth direction at $7.5 \mathrm{rpm}$. Pixel data is quantized into 8 bits via an A/D converter. MIC has lossy image compression capability. An image Compression Module provided by CNES under international collaboration performs JPEG image compression from 3 to 100 times at 4 Mpixels s$^{-1}$ at $14 \mathrm{MHz}$. The compression ratio can be chosen by command from the ground. In standard operation, the imaging area is designated by command as the azimuth start angle, the azimuth image size, the elevation start angle, and the elevation image size. Azimuth angle is defined as the phase angle of the spacecraft rotation whose original point is Sun direction. Elevation angle corresponds to pixel number of the CCD. Image size of each direction can be chosen from 1024, 512 and 256 lines (azimuth) or pixels (elevation). Gain level and image compression parameters are also set by command from the ground.

MIC also has other special onboard functions: "Automatic bright-object imaging" and "Autonomous tracking". The former is realized by the hardware circuits, which search for a pixel brighter than threshold level and then obtains the image 


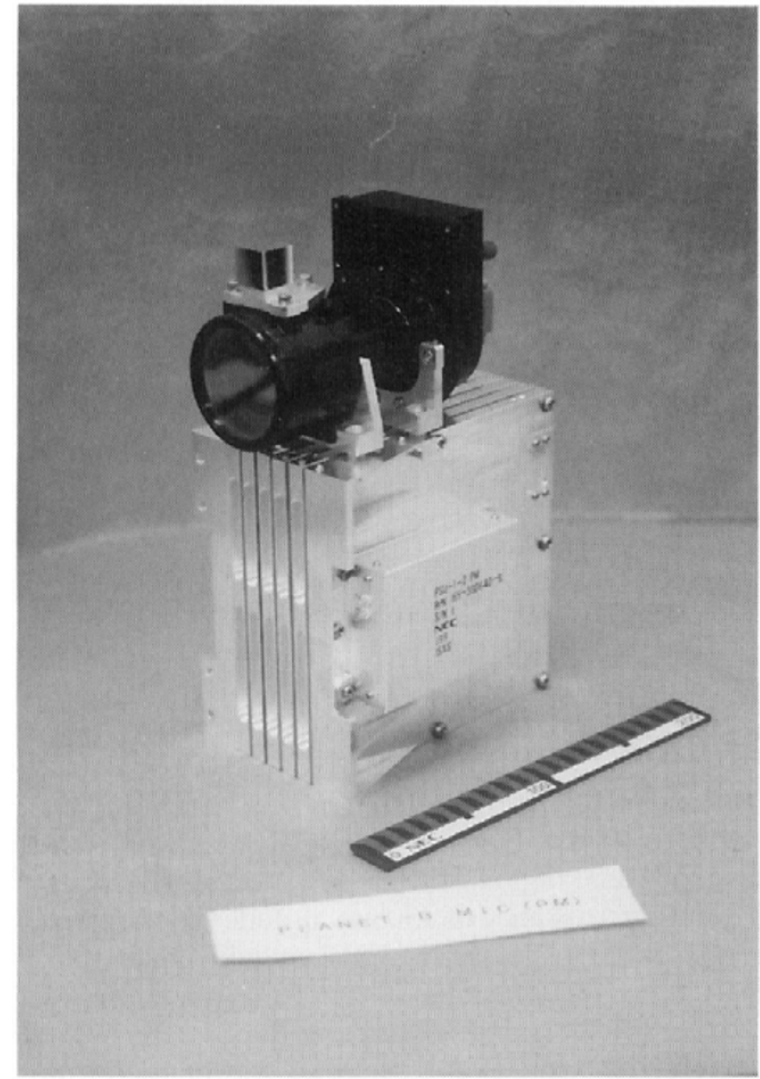

Fig. 1. The Mars Imaging Camera (MIC). Its size is approximately $90 \mathrm{~mm} \times 150 \mathrm{~mm} \times 253 \mathrm{~mm}$.

of neighboring area. Its purpose is for easy operation of Mars global imaging from apoapsis. The latter is realized by the onboard software, which finds the direction of the center of the object, calculates the orbital motion of it, and estimates the direction of it at the next exposure timing. This function is prepared for fly-by imaging of Phobos and Deimos.

\section{Science Objectives}

Since Planet-B takes a very elliptic retrograde orbit, MIC can take global images of Mars. It can monitor changes of global atmospheric conditions on Mars: e.g., dust storm growth, change of atmospheric opacity due to dust and thin hazy clouds, change of cloud features, growth and retreat of polar caps, development and thickness of polar hood. In addition, the planned orbits will make close encounters of Planet-B with Phobos and Deimos several times during the mission lifetime, and consequently, MIC can observe the shapes and the surface structures of two satellites in detail.

When Planet-B is close to Mars near its periapsis, MIC can observe the planetary limb in spatial resolution as high as $2 \mathrm{~km}$, enough for investigating the vertical atmospheric structure. In the following discussion, the main scientific objectives will be discussed based on three broad categories: geology, meteorology, and Martian satellites.

\subsection{Geologic objectives}

Because spatial resolution and data acquisition are limited for MIC, detailed surface mapping cannot be done by MIC as noted above. In some locations, spatial resolution of MIC may exceed that of Viking, i.e. resolution of the image of
MIC, as noted above about 80 arc seconds, yields the spatial resolution of about $60 \mathrm{~m} /$ pixel from a distance of $150 \mathrm{~km}$ altitude (at periapsis of Planet-B). But Mars Global Surveyor will perform detailed morphology mapping prior to PlanetB. However, MIC has many opportunities to obtain images at low solar angle illumination where small undulations are emphasized by longer shadow zones. Moreover, using spacecraft spin, MIC can obtain images in oblique view although it is not equipped with a gimbal mechanism. This may allow MIC to take oblique and sometimes stereographic surface images around periapsis.

The following are examples of the proposed observation plan: characterization of rampart craters and terrain softening as a key for underground water distribution, volcanic and hydrothermal study on Arsia Mons $\left(121^{\circ} \mathrm{W}, 9^{\circ} \mathrm{S}\right)$, stereographic pictures of canyon systems, and relation between aeolean morphology and present wind system.

Figure 2 shows MIC surface resolution, plotted from a compilation of many expected orbits of Planet-B. Planet-B's orbital period is about 34 hours, and subsequently the longitude on Mars during periapsis shifts about $150^{\circ}$ with each successive orbit. Since uncertainties that affect the orbital elements of Planet-B exist around Mars, we have to note that this map is a very preliminary one.

As shown in Fig. 2, Arsia Mons is the only polygenetic volcano in middle-low latitude of southern hemisphere which will be available in the high resolution images of MIC $(\leq 100$ $\mathrm{m} /$ pixel). Mapping of magnetic fields, by using MGF on Planet-B, associated with the imaging of lava flows around Arsia Mons by MIC will show tectonic features originated by volcanic activity, and clarify their evolution.

\subsection{Meteorologic objectives}

In this section we express unsolved problems which are important to investigate Martian atmospheric phenomena. In mid-spring to mid-summer in the northern hemisphere of Mars, the equatorial region is surrounded by a cloud belt extending from $10^{\circ} \mathrm{S}$ to $30^{\circ} \mathrm{N}$ latitudes (Clancy et al., 1996). In the Tharsis area $\left(125^{\circ}-101^{\circ} \mathrm{W}, 12^{\circ} \mathrm{S}-16^{\circ} \mathrm{N}\right)$, being a part of the equatorial cloud belt, many bright clouds appear. They are classified into morning, afternoon, and evening clouds. Since high volcano tops are not covered with those clouds, it is estimated that the cloud height is lower than $20 \mathrm{~km}$. The morning clouds disappear near noon, and simultaneously the afternoon clouds appear. They have a tendency to grow on the huge volcanoes. The afternoon clouds stand out until about Martian local time of 15:00, and get mixed with evening clouds. However, detailed characteristics of these clouds are not yet known. There is a possibility that morning clouds could be derived from moisture trapped in the surface regolith. Monitoring those and other regions at twilight, MIC may clarify the occurrence and nature of the phenomena.

Martian clouds are bright and conspicuous in blue. However surface features are rarely obscured by the clouds in red. This fact suggests that the optical thickness of the clouds is not large. In order to estimate the opacity of clouds precisely, we need the knowledge of the scattering phase function of the clouds, the ground albedo and the scattering phase function of the ground. Clancy and Lee (1991) derived from the Viking data the scattering phase functions of Martian cloud and ground. However the accuracy of their 
Ls

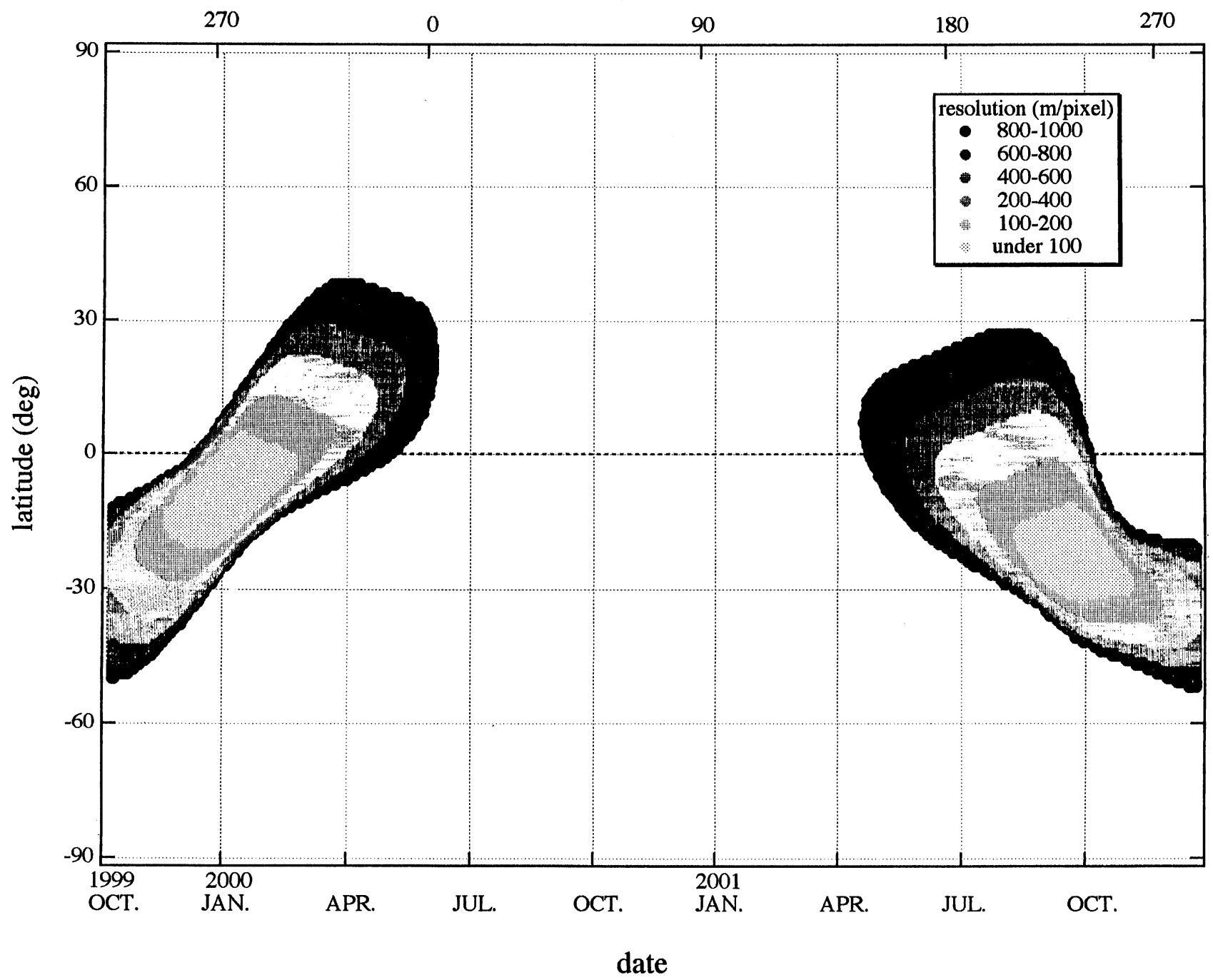

Fig. 2(a). MIC surface resolution, observed during two hours before sunset, during the mission lifetime. $L_{s}$ denotes the areocentric longitude of the Sun. Note that large uncertainties of orbital elements in the later phase of the mission lifetime are expected.

phase functions is restricted to $50^{\circ}$ through $150^{\circ}$ in phase angles. The reflectance of Martian ground is often approximated by Minnaert formula in which Minnaert exponent $k$ is a key parameter (Thorpe, 1977). The value of $k$ is empirically determined as a function of a wavelength and phase angle. Telescopic observations show that $k$ is about 0.7 in red and 0.5 in blue around opposition. Revised analysis of Phobos/KRFM data has provided the value of $k=0.65$ or 0.75 at $550 \mathrm{~nm}$ wavelength (Wuttke et al., 1997). At large phase angles, the apparent Martian disk, seen from the Earth, is too small to determine $k$ precisely. However the images of MIC observations obtained at various phase angles will become a powerful tool to study the surface roughness of the Martian ground, and subsequently to estimate the Minnaert exponent $k$.

Martian cloud features taken by Mariner 9 and Viking Orbiters are classified and their global distribution is described by French et al. (1981), followed by the study considering their seasonal distribution by Kahn (1984). There are lee wave clouds (with an obstacle), wave clouds (with no obstacle), plumes (elongated clouds with sharp edges), streak clouds (elongated clouds without sharp edges), cloud streets (which have two-dimensional periodicity), localized fogs, and hazes. These clouds are basically condensed water ice, but dust as well as ice is responsible for some features, e.g. plumes, streak clouds, fogs, and hazes.

Changes of lee wave clouds which are associated with high mountains and crater rims are one of the most interesting targets for MIC. Using oblique (and sometimes stereographic) views by MIC, we can obtain information on the height and vertical structure of clouds. Lee wave clouds give information on atmospheric gravity waves caused by surface morphology. These gravity waves are transferred upward to the upper atmosphere. Resulting momentum transport may affect the global upper atmosphere circulation. Using observed data of the upper atmosphere by other instruments (e.g., UVS, radio science, NMS) of Planet-B, we can investigate interaction between the lower and upper atmospheres 


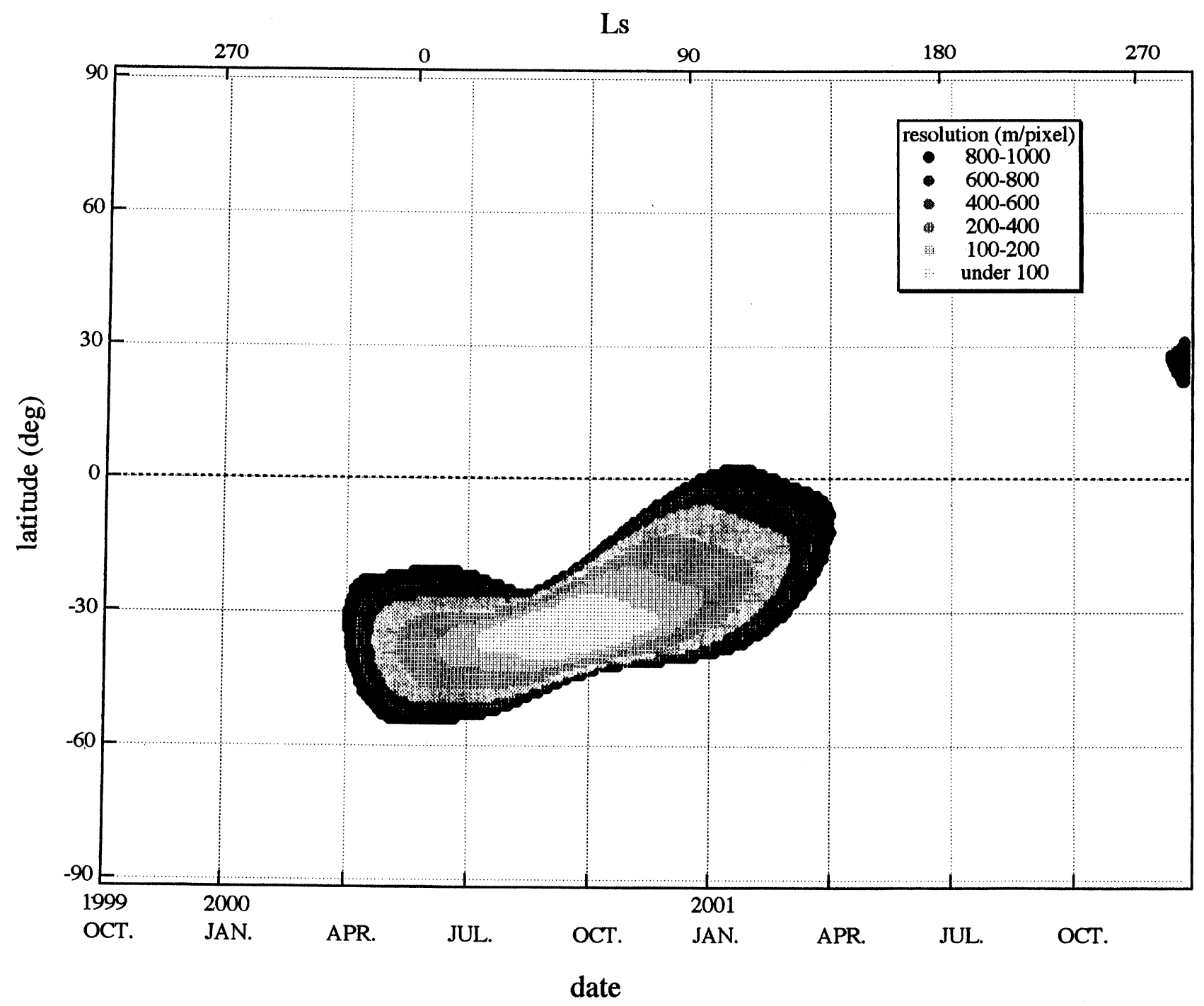

Fig. 2(b). The same as Fig. 2(a), but that, observed during two hours after sunrise.

of Mars.

According to Viking Orbiter observation, there is a continuous dust haze at $10 \sim 30 \mathrm{~km}$ altitude even during no dust storm periods (Jaquin et al., 1986). The optical depth of this haze is $0.1 \sim 0.2$. From wave structure of the haze in limb imaging, we may directly obtain information for wavelength and amplitude of atmospheric gravity waves. Moreover, vertical structure of the haze would give information for meridional circulation as well as turbulence intensity. There is also a thinner detached haze at higher altitude $(30 \sim 90 \mathrm{~km})$. Since the detached haze is condensed water ice, the water vapor mixing ratio in the atmosphere can be obtained from its bottom height. To investigate the vertical and horizontal structure of the atmospheric hazes, MIC should observe the scattering of solar light at the limb. When Planet-B is close to Mars, MIC can observe the planetary limb in spatial resolution as high as $2 \mathrm{~km}$, enough for investigating the vertical structure of Martian atmosphere with atmospheric scale height of $10.8 \mathrm{~km}$.

The most interesting phenomenon in the Martian atmo- sphere is the global dust storms. In the past, they appeared in the southern mid latitudes: Noachis $(1956,1971)$ and Solis Lacus $(1973,1977)$. It is believed that dust is supplied from the original source in a growing stage. However observations from the ground in the 1973 dust storm showed that some areas in the mid latitudes such as Phaethontis and Hellas turned bright in red as the dust cloud moved west (Akabane et al., 1992). It suggests that local or secondary dust storms were induced and they contributed dust to the global cloud. It is one of key scientific objectives of MIC to follow dust storms from their birth to death.

The polar regions in winter have not yet been observed fully from the ground, because the subsolar and subearth points lie far away from the equator in the summer hemisphere. Only the behavior of the north polar hood in autumn have been investigated with the telescopic observations (Akabane et al., 1995). It is reported from the ground-based observations that the bright polar cap appears suddenly in late winter or early spring, until that time we cannot distinguish the polar cap from the polar hood. The process of growing 
of the polar cap has not yet been observed. According to the atmospheric pressure curve obtained by Viking Landers (Hess et al., 1977), the south polar cap may start to grow in late autumn, and the north polar cap may do near winter solstice. MIC will monitor the behavior of the polar hood and polar cap in winter to investigate the polar meteorology.

Mars Global Surveyor (MGS) has a wide angle camera with $140^{\circ}$ field of view (see, e.g. Malin et al., 1992). It can image surface and atmospheric features at moderate resolution (260 $\mathrm{m}$ at nadir and $2 \mathrm{~km}$ at limb) and monitor their changes in time. Although nominal mapping phase of MGS will end in January, 2000, it will monitor Mars during later relay operations if the budget permits. Then, we can compare images of Mars monitoring taken by Planet-B with those by MGS, and also with ground-based and HST observations.

\subsection{Martian satellite objectives}

From the Viking/Phobos images, the following evidence have been found about the groove structure on Phobos surface, i.e., (1) most of the grooves are on the sub-Mars side of Phobos, (2) the grooves appear parallel along the subMars and anti-Mars meridian, and (3) there is a zone with no grooves centered at the trailing apex of Phobos. Furthermore, these grooves seem as if they extend from Stickney, the largest crater on Phobos. The grooves are widest $(700 \mathrm{~m}$ ) and deepest $(90 \mathrm{~m})$ close to the crater itself.

Fujiwara (1991) studied the formation of Stickney, and concluded that the groove structure on Phobos can be explain as crater-associated fractures. Another mechanism proposed to produce the grooves is crater chains (see the review by Thomas et al., 1992). Murray et al. (1994) reported the existence of depressions in these grooves. Moreover, they explained the above features of (1)-(3), assuming the crater chain mechanism associated with the secondary impacts of Martian impact ejecta. To understand the origin of the groove structure on the surface of Phobos, high resolution images of the leading area of Phobos are necessary, where Viking/Phobos could acquire only low resolution images. If the grooves are the fractures of Stickney forming, the same groove structure will exist in the sub-Mars side of Phobos. On the other hand, a lot of intersections of grooves will be seen if they are the crater chains of the secondary impacts of Martian impact ejecta. Furthermore, the crater chain mechanism predicts the existence of the groove structure on Deimos as well as on Phobos, although the number of grooves on Deimos would be less than that on Phobos. MIC observation of Phobos/Deimos during the close encounters will provide important information to solve the question about the origin of grooves.

The existence of Phobos/Deimos dust rings have been predicted theoretically by several authors (e.g., Juhasz and Horanyi, 1995; Hamilton, 1996; Ishimoto, 1996; Krivov and Hamilton, 1997). Many uncertainties still remain in the mechanism of their formation as well as the size/velocity distribution of impact ejecta produced by the interplanetary meteoroids' impacts with these satellites and the validity of self-sustained mechanism for the dust rings. Therefore, there is a large difference up to 6 order of magnitude among the values estimated for the number density of dust particles in the rings. When we apply the largest value of number density, the resulting optical depth of these dust ring becomes about
$10^{-5}$, which is slightly less than the detection limit of Viking imaging camera (Duxbury and Ocampo, 1988), which could not find any sign of dust ring structure.

MIC is designed to detect the surface of the two Martian satellites as its minimum brightness objects, and furthermore, only one shot with the exposure time of $0.49 \mathrm{~ms}$ is available per one rotation of the spacecraft with a speed of $7.5 \mathrm{rpm}$. As the result, MIC is not favourable to see faint objects such as dust rings. However, a significant signal from the hypothetical dust rings might be expected when MIC is placed in the forward scattering light against the dust rings. Namely, the minimum scattering angle predicted during the mission of Planet-B is about $20^{\circ}$. This condition is achieved almost at the end of the Planet-B mission (Oct. 2001). The scattered light flux from dust rings in that occasion is estimated about $5 \mu \mathrm{J} \mathrm{s}^{-1} \mathrm{str}^{-1} \mathrm{~cm}^{-2} \mu^{-1}$. Since the detection limit of MIC is close to this value, we plan to co-add the intensity of several images for the dust rings to enhance the signals.

It should be noted that the number density of the dust rings and its resulting optical depth presented above are the averaged values of the dust particles escaped from the gravity field of the satellite during a fairly long time. A possibility of the existence of relatively dense fresh dust clouds may exist, especially around Phobos and Deimos.

\section{Summary}

MIC observations will be backed up by the ground-based observations via a network of telescopes, organized by Hida Observatory and Nishiharima Astronomical Observatory, throughout Japan. Ground observations will monitor the global conditions on Mars during the mission lifetime. When unexpected events, such as dust storms, are found, urgent messages from the ground-observation network will play a key role in replacing the daily observation plan for MIC immediately.

MIC has some limitations, such as no gimbal mechanism, low rate of data transmission and short available time for MIC during telecommunication. That is, the nominal bit rate is $2 \mathrm{kbps}$. During the time shared for MIC per one revolution of Planet-B around Mars, about 10 compression images with $512 \times 512$ pixels can be received at Earth. However, we hope MIC's unique capabilities described above will provide opportunities for new research and new discoveries in Martian sciences.

Acknowledgments. We thank Juro Ishida for providing the original figure 1 used in this draft.

\section{References}

Akabane, T., K. Iwasaki, Y. Saito, and Y. Narumi, Opacities of the 1973 dust storm over the Solis Lacus, Hellas, and Syrtis Major areas of Mars, Astron. Astrophys., 255, 377-382, 1992.

Akabane, T., Y. Saito, K. Iwasaki, and L. J. Martin, The north polar hood in early autumn on Mars, Astron. Astrophys., 304, 595-601, 1995.

Clancy, R. T. and S. W. Lee, A new look at dust and clouds in the Mars atmosphere-Analysis of emission-phase-function sequences from global Viking IRTM observations, Icarus, 93, 135-158, 1991.

Clancy, R. T., W. Grossman, M. J. Wolff, P. B. James, D. B. Rudy, Y. N Billawala, B. J. Sandor, S. W. Lee, and D. O. Muhleman, Water vapor saturation at low altitudes around Mars Aphelion: A key to Mars climate?, Icarus, 122, 36-62, 1996.

Duxbury, T. C. and A. C. Ocampo, Mars-satellite and ring search from Viking, Icarus, 76, 160-162, 1988. 
French, R. G., P. G. Gierasch, B. D. Popp, and R. J. Yerdon, Global patterns in cloud forms on Mars, Icarus, 45, 468-493, 1981.

Fujiwara, A., Stickney-forming impact on Phobos-Crater shape and induced stress distribution, Icarus, 89, 384-391, 1991.

Hamilton, D. P., The asymmetric time-variable rings of Mars, Icarus, 119, 153-172, 1996.

Hess, S. L., R. M. Henry, C. B. Leovy, J. A. Ryan, and J. E. Tillman, Meteorological results from the surface of Mars-Viking 1 and 2, J. Geophys. Res., 82, 4559-4574, 1977.

Ishimoto, H., Formation of Phobos/Deimos dust rings, Icarus, 122, 153 165, 1996.

Jaquin, F., P. Gierasch, and R. Kahn, The vertical structure of limb hazes in the Martian atmosphere, Icarus, 68, 442-461, 1986.

Juhasz, A. and M. Horanyi, Dust torus around Mars, J. Geophys. Res., 100, 3277-3284, 1995.

Kahn, R., The spatial and seasonal distribution of Martian clouds and some meteorological implications, J. Geophys. Res., 89, 6671-6688, 1984.

Krivov, A. V. and D. P. Hamilton, Martian dust belts: Waiting for discovery, Icarus, 128, 335-353, 1997.

Malin, M. C., G. E. Danielson, A. P. Ingersoll, H. Masursky, J. Veverka,
M. A. Ravine, and T. A. Soulanille, Mars Observer Camera, J. Geophys. Res., 97, 7699-7718, 1992.

Murray, J. B., D. A. Rothery, G. D. Thornhill, J.-P. Muller, J. C. Iliffe, T. Day, and A. C. Cook, The origin of Phobos' grooves and crater chains, Planet. Space Sci., 42, 519-526, 1994.

Thomas, P., J. Veverka, J. Bell, J. Lunine, and D. Cruikshank, Satellites of Mars- Geologic history, in Mars, edited by H. H. Kieffer, B. M. Jakosky, C. W. Snyder, and M. S. Matthews, pp. 1257-1282, Univ. of Arizona, Tucson, 1992.

Thorpe, T. E., Viking orbiter photometric observations of the Mars phase function July through November 1976, J. Geophys. Res., 82, 4161-4165, 1977.

Wuttke, M. W., H. U. Keller, W. J. Markiewicz, E. Petrova, K. Richter, and N. Thomas, Properties of dust in the Mars atmosphere: a revised analysis of Phobos/KRFM data, Planet. Space Sci., 45, 281-288, 1997.

T. Mukai (e-mail: mukai@kobe-u.ac.jp), T. Akabane, T. Hashimoto, H. Ishimoto, S. Sasaki, A. Inada, A. Toigo, M. Nakamura, Y. Abe, K. Kurita, and $\mathrm{T}$. Imamura 